\title{
Study of the Effect of Composite Probiotics on the Intestinal Flora and Serum Globulins of Chicken Meat
}

\author{
Chen Yanli \\ National University Of The Inner Mongol, College of animal science and technology, Inner \\ Mongolia, Tongliao City, 028042 \\ wangzai8402@163.com
}

Keywords: Meat chicken, Bacillus, Lactobacillus

\begin{abstract}
In this study, the feeding effects of chickens with composite probiotics composed with different proportion of Bacillus and lactobacillus were investigated. One hundred and twenty meat chickens were chosen as the experimental subject, and they were feed with composite probiotics composed with different proportion of Bacillus and lactobacillus, and the intestinal flora and serum globulins were detected before and after feeding. The result shows that the bacillus and lactobacillus can improve the immunity of the chicken and regulate the intestinal flora as well as improve utilization rate of feed. But non-significant in improving the survival rate $(\mathrm{P}<0.05)$. The effect of changing the community structure and increasing the serum globulin are significant.
\end{abstract}

\section{Introduction}

The lactic acid bacteria are the dominant fungi of intestinal flora, and the acidophilus lactobacillus is one of the most common ${ }^{\mathbf{2} 2}$. The bacteriocin produced by the acidophilus lactobacillus has direct inhibition function to the bacteria. It can inhibit pathogenic bacteria effectively and occupy the target cell, to form the biological protective barrier ${ }^{\text {[31 }}$ through the colonization competition. The bacillus is aerobic bacteria, and the colonization in the gut can produce the anaerobic environment through the oxygen expenditure. The anaerobic environment promotes the growth of beneficial anaerobic bacteria, and inhibits pathogenic aerobic bacteria invasion and grow at the same time ${ }^{\mathbf{4} \mathbf{I}}$. Most of the bacteria are the anaerobic bacteria in the gut, for example lactobacillus and bifidobacterium and so on. The advantage bacterium group significantly reduce during the diseases such as diarrhea and then the pathogenic microorganism will multiply rapidly. The addition of the microecologics can help the microecosystem in the gut back into balance, and promote the growth and the proliferation of the advantage flora. The result of this experiment shows that the lactobacillus and colon bacillus among the experimental group are improved compared with the contrast group. It means that the compound probiotics can improve the intestinal flora positively. In this study, the feeding effects of chickens with composite probiotics composed with different proportion of Bacillus and lactobacillus were investigated. One hundred and twenty meat chickens were chosen as the experimental subject, and they were feed with composite probiotics composed with different proportion of Bacillus and lactobacillus, and the intestinal flora and serum globulins were detected before and after feeding. 


\section{Materials and Methods}

\section{Design of experiment}

Choosing one hundred and twenty meat chickens as the experimental subject, and randomly divided the chickens into four groups. Each group had three repetitions, 10 chickens in each repetition. The control group feed on basal diet. Among the experimental groups, group one add $0.1 \%$ bacillus $+0.3 \%$ lactobacillus in the basal diet, group two add $0.2 \%$ bacillus $+0.2 \%$ lactobacillus in the basal diet, group three add $0.3 \%$ bacillus $+0.1 \%$ lactobacillus in the basal diet.

Bacterial strain

Bacillus cssrcus and Lactobaoillus acidophilus are provided by the microbiology laboratory of animal science and technology College. The Bacillus cssrcus use the aerobic culture method and shock cultured with $37^{\circ} \mathrm{C}$ on the shaking table for $48 \mathrm{~h}$ and the content of bacteria is $11 \times 10^{8}$ per $\mathrm{mL}$. The Lactobaoillus acidophilus use the anaerobic culture method with $37^{\circ} \mathrm{C}$ in the $2000 \mathrm{ml}$ tubulated bottle for $48 \mathrm{~h}$ and the content of bacteria is $9 \times 10^{10}$ per $\mathrm{mL}$.

Preparation methods of biological food additives: Mix the bacterial suspension with $4.5 \%$ Sodium alginate solution in the capacity ratio of $1: 50$ with $37^{\circ} \mathrm{C}$, then drop the mixed suspension into the $2 \% \mathrm{CaCl}_{2}$ solution to get the gel balls containing the bacteria. Finally mix the balls with the basal diet according to a certain proportion.

The basal diet composition and the nutritional level

The basal diet is the corn with soybean powder, the preparation method according to the

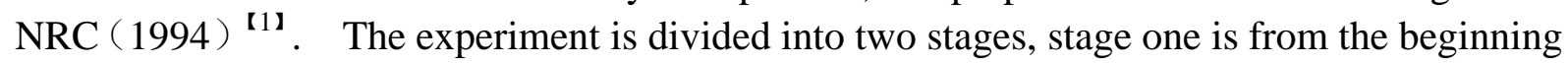
to 21 days and stage two from 22 days to 42 days. Table 1 is the component and the nutritional level of the basal diet. The preparation method of the experimental diet: To mix the basal diet with the probiotics in the proportion of 10:1 to get the premix feed, then mix the premix feed with the residual basal feed thoroughly. To ensure the stability of the viable quantity, the mixed feed should be stirred every three days.

Feeding Management

Using the flat culture on the entanglement, provide the 24hours illumination, gathering the food and water freely. The relative humidity in the cage is $60 \% \pm 5 \%$. During the experimental period, the use of any antimicrobial drugs and additives should avoid, and other management according to the routine.

Sample collection and index testing

At the beginning of the experiment, weighting all the samples in every group, making recordings and inspections. The original weight difference of each chicken should not too significant. Measuring the consumption of feed at 21days and 42days, as well as the final weight of the samples in the morning of these two days. Keeping a record of the health condition as well as the death numbers. Calculating the survival rate, gain weight and the feed conversion ratio. After the weighting, choose a sample from each repeating group, and make sure each experimental group have two hens and one cock. Taking samples of blood from each chicken. In 21days using the wing vein or jugular vein blood sampling method and the 
heart blood sampling method was used in 42days. The blood capacity taken is $5 \mathrm{ml}$ from each chicken and divided into three test tubes. Slant setting the tubes into the thermostat with the temperature of $38^{\circ} \mathrm{C}$, and centrifuge the blood at the speed of $3000 \mathrm{r} / \mathrm{min}$ to separate the serum. Put the serum into the refrigerator at the temperature of $-4^{\circ} \mathrm{C}$ for the later use. After all the processes mentioned above finished, kill the samples and separate the jejunum, put $1 \mathrm{~g}$ jejunum into the test tube and analyze the microorganism.

Assay method

Using the plate count method to analyze the microorganism. Measuring the $\gamma$-globulin by cellulose acetate membrane electrophoresis.

Table 1 Basal diet component and the nutritional level

\begin{tabular}{lcc}
\hline & $22 \mathrm{~d}$ & $22-42 \mathrm{~d}$ \\
\hline Basal diet & & \\
Corn (\%) & 61.65 & 64.77 \\
Bean pulp (\%) & 26.10 & 24.79 \\
Fish meal (\%) & 6.00 & 4.00 \\
DCP (\%) & 0.95 & 1.38 \\
Stone powder (\%) & 0.80 & 0.65 \\
Vegetable oil (\%) & 1.00 & 0.86 \\
Salt (\%) & 0.20 & 0.25 \\
Bone powder (\%) & 3.30 & 3.00 \\
premix (\%) & 0.30 & 0.30 \\
Nutritional level & & \\
Metabolic & 13.00 & 13.00 \\
Crude protein (\%) & 22.14 & 19.97 \\
calcium (\%) & 0.93 & 0.96 \\
Total phosphorus & 0.68 & 0.66 \\
Available & 0.45 & 0.43 \\
Methionine (\%) & 0.56 & 0.46 \\
Cystine (\%) & 0.33 & 0.32 \\
Lysine (\%) & 0.99 & 0.93 \\
\hline
\end{tabular}

Notes:

(1)Vitamin additives in the premix is Ted multidimensional $165 \mathrm{mg} / \mathrm{kg}$.(2)Crude protein, calcium and total phosphorus are the measured values. Metabolic energy, available phosphorus and the amino acid are the literature value.

Statistic analysis

Using the SAS statistics analysis software to do the variance analysis, and the multiple comparisons use the Duncan's method.

\section{Result and analysis}

The influence on the health condition and the survival ratio of the meat chickens

The data in Table 2 show: To compare the three experimental groups with the contrast group, the improvement of the survival ratio are $4.34 \%, 4.34 \%$ and 0 at the first 
stage(0-21days). At the second stage(21-42days), the improvement of the survival ratio are $6.99 \%, 4.47 \%$ and $6.99 \%$. The improvement of the survival ratio in the full stage are $11.11 \%$, $8.89 \%$ and $6.67 \%$. The chickens grow normally in the whole experimental process. There are two chickens paste the anal in the experimental group three and one in the experimental group two. No chicken in experimental group one have the diarrhea, and experimental group one gets the best result.

Table 2 the effect of the composite probiotics of bacillus and lactobacillus to the survival ratio of the meat chickens

\begin{tabular}{clll}
\hline & $0-21 \mathrm{~d}$ & $22-42 \mathrm{~d}$ & $0-42 \mathrm{~d}$ \\
\hline Contrast group & $95.66 \pm 2.93$ & $93.01 \pm 3.12$ & $88.89 \pm 2.79$ \\
Experimental group one & $100.00 \pm 0.00$ & $100.00 \pm 0.00$ & $100.00 \pm 0.00$ \\
Experimental group two & $100.00 \pm 0.00$ & $97.78 \pm 1.79$ & $97.78 \pm 1.79$ \\
Experimental group three & $95.66 \pm 2.93$ & $100.00 \pm 0.00$ & $95.66 \pm 2.93$ \\
\hline
\end{tabular}

The influence on the intestinal flora of the meat chickens

The data in table 3 show: The number of escherichia coli decreased significantly in every experimental groups. With the exception of the group three,there is a significant difference compared experimental group one and group two with the contrast group $(\mathrm{P}<0.05)$. The ratio of the coliform and lactobacilli are improved except experimental group three $(\mathrm{P}<0.05)$.The effect of experimental group two and three is better.

Table 3 The effect of composite probiotics to the coliform count and lactobacilli number in the jejunum of

42days meat chickens $\left(\log 10^{\mathrm{n}} / \mathrm{g}\right.$ chicken manure $)$

\begin{tabular}{cccc}
\hline Contrast group & $4.47 \pm 0.25$ & $4.58 \pm 0.14$ & $1: 1.02 \pm 0.00$ \\
Experimental group one & $4.11 \pm 0.37$ & $5.51 \pm 0.62$ & $1: 1.35 \pm 0.04$ \\
Experimental group two & $2.54 \pm 0.11^{*}$ & $5.89 \pm 0.38$ & $1: 2.32 \pm 0.02^{*}$ \\
Experimental group three & $3.21 \pm 0.21^{*}$ & $5.69 \pm 0.18$ & $1: 1.77 \pm 0.03^{*}$ \\
\hline
\end{tabular}

The effect on the $r$ serum globulins of the meat chickens

The data in the table 4 show: The improvement of $r-\mathrm{G}$ in every experimental groups are $3.13 \%, 4.15 \%$ and $1.40 \%$ in 22 days, and the differences are not very significant. The experimental group three improved $6.46 \%$ in 42days, the difference with the contrast group is not significant, too. The improvement in the experimental group one and two are $15.81 \%$ and $25.68 \%$, and there is a significant improvement compared with the contrast group. The difference among every experimental group is not very clear and the experimental group one is the most significant group.

Table 4 The effect of composite probiotics to the $r$ serum globulins $(r-G)$ in the meat chickens $(\mathrm{mg} / \mathrm{dl})$ 


\begin{tabular}{lcc}
\hline & $21 \mathrm{~d}$ & $42 \mathrm{~d}$ \\
\hline Contrast group & $98.24 \pm 2.12$ & $104.56 \pm 1.64$ \\
Experimental group one & $101.31 \pm 3.01$ & $121.09 \pm 2.01^{*}$ \\
Experimental group two & $102.32 \pm 3.66$ & $131.41 \pm 1.98^{*}$ \\
Experimental group three & $99.62 \pm 1.39$ & $111.32 \pm 2.11$ \\
\hline
\end{tabular}

\section{Discussion and conclusion}

The compound proboscis can improve the activity of the immune system as well as the synthesis and secretion of the immunoglobulin. The level of the immune globulin in every experimental groups are improved significantly. It proves that the compound probiotics can enhance immune function positively.

In conclusion, The compound probiotics can improve the immune function through improving the balance of the micro-ecology in the digestive tract, and then achieve the effect of disease prevention as well as improve the survival ratio of the meat chickens. No adverse effects were found during the experiment and the compound probiotics is feasible in the poultry Industry.

\section{References}

[1] NRC.Nutrient Requirement of poultry: $9^{\text {th }}$ edition [M].National Academies Press,Washington D C.1994

[2] Fu Yuehua.The separation and application of lactic acid bacteria from the animal gut. Feed Research.1994,(5):11-12

[3] Xu Liping, Song Yunpeng, Lou Yujie. The influence of compound bacterium to the production performance and the normal flora of the meat chickens. Feed Review[J] .2002, (2):29-31

[4] Shen Sufang. Fan Yuan. Ma Yanna. The influence of compound bacterium to the production performance of the meat chickens.China Animal Husbandry \& Veterinary Medicine.2012,(8):23-34 